\title{
Ausgrabungen im Bereich des Murus Gallicus 1990-1993
}

\author{
Kaspar Richner \\ unter Mitarbeit von Eckhard Deschler-Erb und Christian Stegmüller
}

Die Ausgrabungen im Bereich des Murus Gallicus auf dem Basler Münsterhügel in den Jahren 1990-19931 haben zwei Ergebnisse gezeitigt, die auch ausserhalb der lokalen Forschung auf Interesse stossen dürften:

\section{Methode}

Zum einen wurde eine Methode perfektioniert, mit der sich komplexe Holzstrukturen selbst in Trockenböden dokumentieren und zuverlässig dreidimensional rekonstruieren lassen. Diese Methode lässt sich bei Objekten aus jeder beliebigen Epoche anwenden.

Sie besteht im Wesentlichen darin, dass die zerfallenen Holzstrukturen in Abbauschichten abgetragen und dokumentiert werden, die nicht dicker als die einstigen Hölzer sein dürfen, und dass die Hohlräume oder lockeren Zonen am Ort der ursprünglichen Hölzer nach Möglichkeit bereits lokalisiert werden, bevor sie flächig aufgebrochen sind. Dies geschieht von bestehenden Profilen aus oder durch systematisches Absuchen der Abbauflächen mit einer Sonde (Abb. 1). Die zerfallene Holzkonstruktion muss beim erstmaligen Freilegen erkannt werden oder aber bereits von früheren Grabungen bekannt sein, damit rechtzeitig auf diese differenzierte Sondier- und Dokumentationsmethode umgestellt werden kann.

Aus den einzeln dokumentierten Stücken werden bei der Aufarbeitung wieder die ganzen Balken rekonstruiert (Abb. 2); anschliessend werden die einzelnen Balken zur vollständigen dreidimensionalen Holzkonstruktion zusammengefügt. Dies kann zeichnerisch, mit Hilfe eines Modelles (Abb. 3) oder unter Verwendung modernster elektronischer Hilfsmittel erfolgen.

Die Methode ist aufwendig: Sie verlangt ein konstantes Abbautempo (nach jeder Abbauschicht von 10-15 cm Dicke muss ein Grundriss gezeichnet werden) und ebensoviel Zeit für die Auswertung wie für die eigentliche Ausgrabung.

\section{Struktur des Murus Gallicus}

Zum andern wurde, unter Anwendung der oben beschriebenen Methode, die Struktur des Murus Gallicus auf dem Basler Münsterhügel vollständig (aber ohne ungesicherte Ergänzungen) dokumentiert, soweit sie überhaupt noch erhalten war.

Die Front des Walles besteht aus einer Pfostenschlitzmauer; das Wallinnere ist mit Holzkästen (Queranker und Längsbalken in ununterbrochenen Lagen) armiert. Kurze Pfostenanker verbinden die senkrechten Pfosten in der Wallfront mit den Holzkästen des Wall-

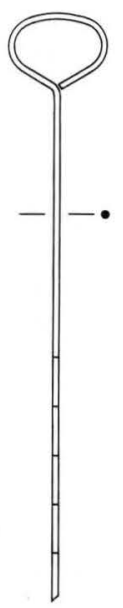

$A b b$. 1. Sonde für die Untersuchung verborgener Hohlräume. - Zeichnung: Ch. Stegmüller. - Massstab 1:20.

innern. Sie sind mit Nägeln an den senkrechten Frontpfosten und an den Holzkästen im Wallinnern befestigt; ebenso sind sämtliche Balkenlagen der Holzkästen im Wallinnern miteinander vernagelt. Kurze Balkenstücke versteifen zusätzlich die Holzkästen unmittelbar hinter der Wallfront. Die Queranker (quer zum Verlauf des Walles) erscheinen in einem Schnitt durch den Wall aufgefächert, d. h. sie sind ca. ab der drittuntersten Lage zur Wallinnenseite hin geneigt. Auf dem Baugrund liegt hinter der Wallfront eine Packung von Bruchsteinen oder Wacken, die das Sickerwasser sammelt und die Holzarmierung im Wallinnern trocken hält. Dadurch bleibt das Mikroklima konstant und die Lebensdauer der Holzkästen verlängert sich.

Der Basler Murus Gallicus ist einphasig ${ }^{2}$; nur die Wallfront wurde an zwei Stellen (zu verschiedenen Zeitpunkten) lokal repariert; die Holzkästen im Wallinnern sind von diesen lokalen Frontreparaturen nicht betroffen.

Die maximal erhaltene Höhe des Murus Gallicus beträgt noch $2 \mathrm{~m}$ im Innern des Walles, im Frontbereich etwa $1 \mathrm{~m}$; der Wall konnte von der Front aus bis in ca. $8 \mathrm{~m}$ Tiefe untersucht werden ${ }^{3}$.

Lässt man bei den früheren Grabungen am Murus Gallicus von 1971-1979 die ungesicherten Ergänzungen ${ }^{4}$ weg und macht die vom Befund her nicht vorgegebene Aufteilung auf zwei Bauphasen im Rahmen einer vermeintlichen Totalerneuerung rückgängig, dann lassen sich die übrigbleibenden, eindeutig festgestellten Elemente problemlos in unser neues Modell integrieren: Sie sind ein - allerdings unvollständiger Ausschnitt aus den heute bekannten Bauelementen. 

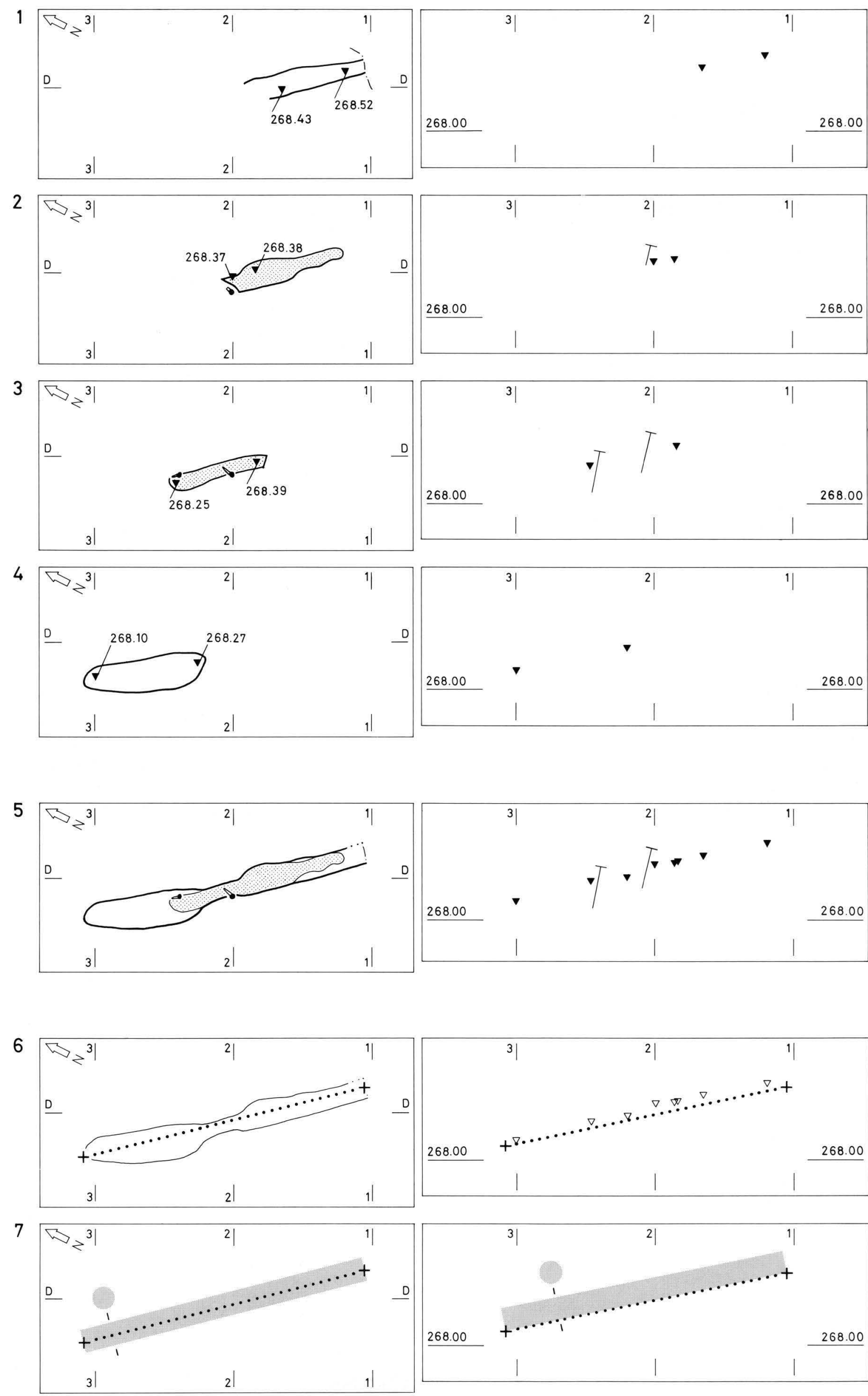

$A B B 2$ 


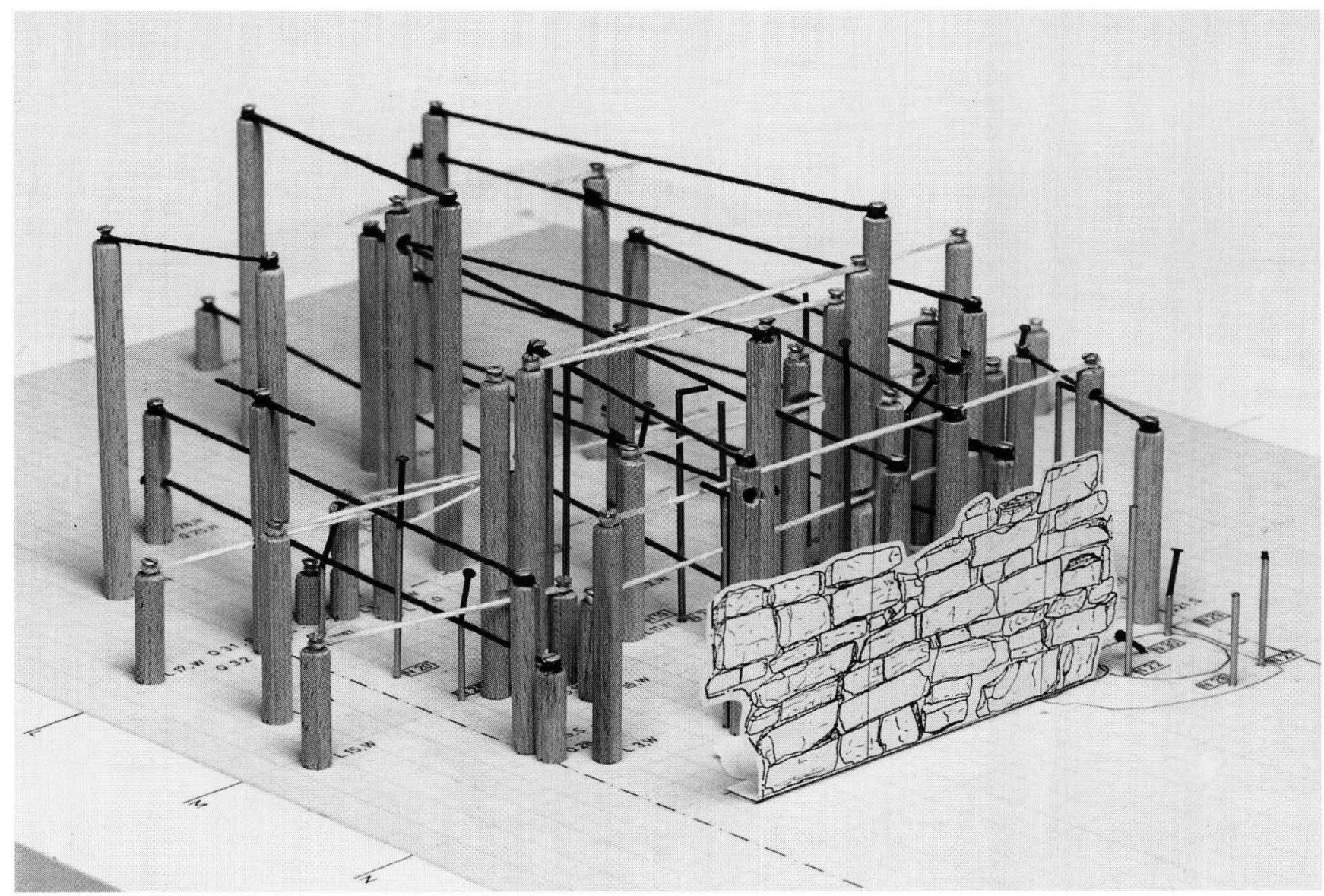

Abb. 3. Modell des Murus Gallicus (Ausschnitt, Sektor 1 +2) mit wiederaufgerichteter Trockenmauer (Tusche auf Karton), Balkengitter (helle und dunkle Wollfäden) und Nägeln (schwarzgefärbte Drahtstifte). Rechts des erhaltenen Teilstückes der Trockenmauer ist auf dem Baugrund die Pfostengrube und der Holzschatten eines senkrechten Frontpfostens markiert (zwei konzentrische Kreise). Die senkrechten Holzstäbe und die senkrechten ungefärbten Drahtstücke sind modellbautechnisch bedingte Stützen und haben nichts mit der Struktur des Walles zu tun. - Modell: S. Böttcher - Photo: Th. Kneubühler. - Maximale erhaltene Höhe der Trockenmauer an der Wallfront ca. $1 \mathrm{~m}$, maximale erhaltene Höhe des Balkengitters ca. $2 \mathrm{~m}$.

Abb. 2. Beispiel für die zeichnerische Rekonstruktion eines Balkens im Grundriss (Abbildungsreihe links) und Profil (Abbildungsreihe rechts) inkl. Aufbereitung für die computerbasierte Visualisierung. - Zeichnung: Ch. Stegmüller. - Massstab 1:40.

\section{Legende:}

1-4 Grabungsdokumentation: links vier aufeinanderfolgende Abbauschichten; rechts jeweils die Projektion der nivellierten Höhenpunkte auf der Sohle des Balkengräbchens (schwarze Dreiecke) ins Profil

5 Rekonstruktion des gesamten erhaltenen Balkens (Überlagerung von "1"-" 4"), mit Nägeln

6-7 Aufbereitung für die computerbasierte Visualisierung

\section{Anmerkungen}

1 Vorbericht: Eckhard Deschler-Erb und Kaspar Richner, „Murus Gallicus, Vorbericht zu den Grabungen 1990 bis 1992“, JbAB 1991 29-33. - Publikation in Vorbereitung, erscheint in der Reihe der Materialhefte zur Archäologie in Basel (ABS).

2 Der Wall kann nur ungefähr in die Mitte oder zweite Hälfte des 1. Jh. v Chr. datiert werden. Der Terminus ante quem wird durch den römischen Horizont über der Wallruine resp. dem Versturz des Murus Gallicus gegeben, der nach Ausweis der Funde um 20 v. Chr.-20 n. Chr. abgelagert wurde

3 Die Innenflucht des Walles wurde bei den Grabungen von 19901993 nicht erreicht; die Gesamtbreite des Walles und die Bauweise der inneren Flucht sind daher nicht bekannt. - Die Dokumentation des Ostschnittes der Grabung 1976 ist in den Teilen, die den inneren Teil des Murus Gallicus betreffen könnten, so summarisch, dass daraus keine gesicherten Erkentnisse entnommen werden können.

4 Andres Furger-Gunti, „Der Murus Gallicus von Basel“, JbSGUF 63, 1980, 131-184. 


\section{Anhang}

\section{Abkürzungen}

AB Archäologische Bodenforschung

BaDpfl. Basler Denkmalpflege

BS Bodenscherbe

FK Fundkomplex

Fl. Fläche

$\mathrm{H} \quad$ Horizont

HGB Historisches Grundbuch

$\mathrm{HMB}$ Historisches Museum Basel

Inv.-Nr. Inventar-Nummer

$\mathrm{Jb} \quad$ Jahresbericht

KMBL Kantonsmuseum Basel-Land

Mk Münzkabinett (HMB)

MR Mauer

MVK Museum für Völkerkunde

NHM Naturhistorisches Museum

OF Oberfläche

OK Oberkante

P Profil

RMA Römermuseum Augst

RS Randscherbe

Sd Sonderdruck

SS Sondierschnitt

StAB(S) Staatsarchiv Basel-Stadt

UK Unterkante

WS Wandscherbe

Literatursigel (Zeitschriften, Reihen etc.)

ABS Archäologie in Basel. Materialhefte zur Archäologie in Basel

AS Archäologie der Schweiz

ASA Anzeiger für Schweizerische Altertumskunde

(B)Njbl. (Basler) Neujahrsblatt. Herausgegeben von der Gesellschaft zur Beförderung des Guten und Gemeinnützigen.

BUB Urkundenbuch der Stadt Basel, Bände 111. Herausgegeben von der Historischen und Antiquarischen Gesellschaft zu Basel, Basel.

BZ Basler Zeitschrift für Geschichte und Altertumskunde

JbAB Jahresbericht der Archäologischen Bodenforschung des Kantons Basel-Stadt

JbAK Jahresberichte aus Augst und Kaiseraugst

JbHMB Jahresbericht des Historischen Museums Basel-Stadt

JbSGUF Jahresbericht der Schweizerischen Gesellschaft für Ur- und Frühgeschichte

KDM BS Die Kunstdenkmäler des Kantons BaselStadt, Bände 1-5. Herausgegeben von der Gesellschaft für Schweizerische Kunstgeschichte, Basel.
NSBV Nachrichten des Schweizerischen Burgenvereins

SBKAM Schweizer Beiträge zur Kulturgeschichte und Archäologie des Mittelalters

ZAK Zeitschrift für Schweizerische Archäologie und Kunstgeschichte

ZAM Zeitschrift für Archäologie des Mittelalters

\section{Schriften der Archäologischen Bodenforschung}

Jahresberichte (JbAB)

Der Jahresbericht 1994 kann, solange vorrätig, zum Preis von Fr. 40.- bei der Archäologischen Bodenforschung bezogen werden. Die Jahresberichte 1988 bis 1993 sind zu Fr. 10.- noch erhältlich.

Materialhefte zur Archäologie in Basel (ABS)

Ergänzend zu den Jahresberichten wird in den Materialheften zur Archäologie in Basel eine repräsentative Auswahl von Basler Fund- und Dokumentationsmaterial vorgelegt. Mit der Schriftenreihe soll die abschliessende Berichterstattung über eine Grabung mit nachvollziehbarer Beweisführung und Auswertung des Fundmaterials ermöglicht werden.

Bisher erschienen und solange vorrätig noch erhältlich

Rudolf Moosbrugger-Leu, Die Chrischonakirche von Bettingen. Archäologische Untersuchungen und baugeschichtliche Auswertung. Mit einem Beitrag von Beatrice Schärli über die Münzfunde. Verlag Archäologische Bodenforschung Basel-Stadt, Basel 1985. Materialhefte zur Archäologie in Basel, Heft 1. 110 Textseiten, 78 Abbildungen, 6 Fototafeln und 3 Faltpläne. ISBN 3-905098-00-8. Fr. 15.-

Rudolf Moosbrugger-Leu, Peter Eggenberger, Werner Stöckli, Die Predigerkirche in Basel. Mit einem Beitrag von Beatrice Schärli über die Münzfunde. Verlag Archäologische Bodenforschung Basel-Stadt, Basel 1985. Materialhefte zur Archäologie in Basel, Heft 2. 133 Textseiten, 108 Abbildungen, 5 Faltpläne. ISBN 3-905098-01-6. Fr. 15.-

Peter Thommen, Die Kirchenburg von Riehen. Mit Beiträgen von Kurt Wechsler und Marcel Mundschin. Verlag Archäologische Bodenforschung Basel-Stadt, Basel 1993. Materialhefte zur Archäologie in Basel, Heft 5. 172 Textseiten, 135 Abbildungen, 15 Tafeln. ISBN 3905098-08-3. Fr. 40.-.

Thomas Maeglin, Spätkeltische Funde von der Augustinergasse in Basel. Mit einem osteologischen Beitrag von Jörg Schibler. Verlag Archäologische Bodenforschung Basel-Stadt, Basel 1986. Materialhefte zur Archäologie in Basel, Heft 6. 97 Textseiten, 33 Abbildungen, 14 Tafeln. ISBN 3-905098-02-4. Fr. 15.-

Dieter Holstein, Die bronzezeitlichen Funde aus dem Kanton BaselStadt. Verlag Archäologische Bodenforschung Basel-Stadt, Basel 1991. Materialhefte zur Archäologie in Basel, Heft 7. 95 Textseiten, 17 Abbildungen, 36 Tafeln, 1 Faltplan. ISBN 3-905098-09-1. Fr. 15.-.

Jacqueline Reich, Archäozoologische Auswertung des mittelalterlichen Tierknochenmaterials (10.-13. Jh.) von der Schneidergasse 8, 10 und 12 in Basel ( $\mathrm{CH}$ ). Mit einem Beitrag von Christoph Ph. Matt. 
Verlag Archäologische Bodenforschung des Kantons Basel-Stadt, Basel 1995. Materialhefte zur Archäologie in Basel, Heft 8. 84 Textseiten, 75 Abbildungen, 74 Tabellen. ISBN 3-905098-15-6. Fr. 45.-.

René Matteotti, Die Alte Landvogtei in Riehen. Ein archäologischer Beitrag zum Alltagsgerät derNeuzeit. Verlag Archäologische Bodenforschung des Kantons-Basel-Stadt, Basel 1994. Materialhefte zur Archäologie in Basel, Heft 9. 82 Textseiten, 56 Abbildungen, 33 Tafeln, 2 Farbtafeln. ISBN 3-905098-14-8. Fr. 40.-.

Pia Kamber, Die Latrinen auf dem Areal des Augustinerklosters, Basel-Augustinergasse 2, Grabung 1968. Mit einem Beitrag von F. Maurer zur Baugeschichte des Klosters. Weitere Beiträge von S. Jacomet (Archäobotanik), M. Joos (Sedimentologie), J. Schibler (Archäozoologie) und W.B. Stern (Archäometrie). Verlag Archäologische Bodenforschung des Kantons Basel-Stadt, Basel 1995. Materialhefte zur Archäologie in Basel, Heft 10. 153 Textseiten, 111 Abbildungen, 52 Tafeln, 5 Farbtafeln, 1 Falttafel. ISBN 3-905098-17-2. Fr. 45.-.

Marlu Kühn, Spätmittelalterliche Getreidefunde aus einer Brandschicht des Basler Rosshof-Areales (15. Jahrhundert AD). Verlag Archäologische Bodenforschung des Kantons Basel-Stadt, Basel 1996. Materialhefte zur Archäologie in Basel, Heft 11.78 Textseiten, 43 Abbildungen/ Diagramme, 87 Zeichnungen, 19 Tabellen. ISBN3-905098-19-9. Fr.45.-

Weitere Veröffentlichungen der Archäologischen Bodenforschung des Kantons Basel-Stadt

Rolf d'Aujourd'hui, Archäologie in Basel. Fundstellenregister und Literaturverzeichnis. Jubiläumsheft zum 25jährigen Bestehen der Archäologischen Bodenforschung Basel-Stadt. Herausgegeben von der Archäologischen Bodenforschung Basel-Stadt mit Unterstützung der Historischen und Antiquarischen Gesellschaft zu Basel. Verlag Archäologische Bodenforschung Basel-Stadt, Basel 1988. 179 Seiten, 5 Abbildungen. ISBN 3-905098-04-0. Fr. 10.-

Rolf d'Aujourd'hui, Die Entwicklung Basels vom keltischen Oppidum zur hochmittelalterlichen Stadt. Überblick Forschungsstand 1989. Zweite überarbeitete Auflage. Verlag Archäologische Bodenforschung Basel-Stadt, Basel 1990. 25 Textseiten, 35 Abbildungen. ISBN 3905098-05-9. Fr. 10.-. (Zur Zeit vergriffen, Neuauflage in Vorbereitung.)

Rolf d'Aujourd'hui, Basel Leonhardsgraben 47: Eine Informationsstelle über die mittelalterliche Stadtbefestigung im Teufelhof, Führer zur Ausstellung, Sd aus: Unsere Kunstdenkmäler 41, 1990.2, 169-180. Fr. 3.-
Rolf d'Aujourd'hui, Der Archäologische Park am Murus Gallicus, Führer durch die Ausstellung an der Rittergasse in Basel, Sd aus: Basler Stadtbuch 1993, 196-204. Fr. 3.-.

Ulrike Giesler-Müller, Das frühmittelalterliche Gräberfeld BaselKleinhüningen. Katalog und Tafeln. Basler Beiträge zur Ur- und Frühgeschichte, Bd. 11 B. Habegger Verlag, Derendingen-Solothurn 1992 221 Textseiten, 113 Tafeln, 1 Faltplan. ISBN3-85723-321-4. (Nur über Buchhandelerhältlich.)

Peter Jud (Hrsg.), Die spätkeltische Zeit am südlichen Oberrhein/Le Rhin supérieur à la fin de l'époque celtique, Kolloquium Basel, 17./18. Oktober 1991/Colloque de Bâle, 17/18 octobre 1991. Zweite, unveränderte Auflage. Verlag Archäologische Bodenforschung des Kantons Basel-Stadt, Basel 1995. 179 Seiten, zahlreiche Abbildungen. ISBN 3-905098-13-X. Fr. 40.-.

\section{Bestellmöglichkeiten}

Die Hefte werden von der Archäologischen Bodenforschung und vom Seminar für Ur- und Frühgeschichte der Universität Basel im Selbstverlag herausgegeben und sind über den Buchhandel oder beim Verlag direkt erhältlich. Bestellungen sind zu richten an: Archäologische Bodenforschung Basel-Stadt, Petersgraben 11, 4051 Basel.

Einzelbestellung. Es gelten die oben erwähnten Preise zuzüglich Versandkosten.

Abonnement Materialhefte. Der Preis je Heft beträgt Fr. 35.- zuzüglich Versandkosten. Die Auslieferung erfolgt jeweils nach Erscheinen eines Heftes.

Abonnement Jahresbericht. Der Preis je Jahrgang beträgt Fr. 30.- zuzüglich Versandkosten.

Wenn Sie Jahresbericht und Materialheft abonniert haben, gewähren wir Ihnen einen Rabatt von Fr. 10.auf den Abonnements-Preis des Jahresberichtes. 